\title{
An analysis of the durations of Swift gamma-ray bursts
}

\author{
Z.-B. Zhang ${ }^{1,2}$ and C.-S. Choi ${ }^{1}$ \\ 1 International Center for Astrophysics, Korea Astronomy and Space Science Institute, 36-1 Hwaam, Yusong, Daejon 305-348, \\ South Korea \\ e-mail: zbzhang@kasi.re.kr \\ 2 Yunnan Observatory, National Astronomical Observatories, Chinese Academy of Sciences, PO Box 110, Kunming 650011, \\ PR China \\ Received 7 December 2007 / Accepted 5 March 2008
}

\begin{abstract}
Context. Swift detectors are found to be more sensitive to long-soft bursts than pre-Swift missions. This may largely bias the distribution of durations and thus classification of gamma-ray bursts.

Aims. We systematically investigate the duration distribution of gamma-ray bursts in the Swift era vs. that of pre-Swift bursts.

Methods. For the purpose of this study, statistical methods such as the K-S test and linear/non-linear fitting analysis have been used to examine the duration properties of Swift bursts in both observer and source frames.

Results. For 95 GRBs with known redshift, we show that two log-normal distributions of duration are clearly divided at $T_{90} \simeq 2 \mathrm{~s}$. The intrinsic durations also show a bimodal distribution but shift systematically toward the smaller value and the distribution exhibits a narrower width than the observed one. Swift long bursts exhibit a wider duration dynamic range in both observer and source frames in comparison to pre-Swift long bursts.

Conclusions. We find that Swift bursts and pre-Swift ones can share the same criterion of classification in terms of duration at $2 \mathrm{~s}$, although both monitors have large differences with respect to sensitivity of a given energy band.
\end{abstract}

Key words. gamma rays: bursts - gamma rays: theory

\section{Introduction}

Cosmic gamma-ray bursts (GRBs) are the most violent explosions occurring at cosmological distances in the universe. When a GRB takes place, satellites can monitor its temporal variability in the $\gamma$-ray energy band. The duration of the burst, $T_{90}$, is defined as the time interval in which the integrated photon counts increase from $5 \%$ to $95 \%$ of the total counts. Based on an analysis of durations using initial BASTE data, Kouveliotou et al. (1993) divided GRBs into two classes, i.e., long GRBs (LGRBs) with $T_{90}>2$ s and short GRBs (SGRBs) with $T_{90}<2$ s. The dichotomy has been justified by subsequent investigations (e.g., Mao et al. 1994; Katz \& Canel 1996; Meegan et al. 1996; Paciesas et al. 1999; Fishman 1999). The best parameters of a two-component log-normal fit to the distribution data were first obtained by McBreen et al. (1994). This fit is supported by the current BATSE data with peak flux information (Horváth 2002; Nakar 2007).

So far, much evidence showing the difference between two classes has been discovered and presented (see Zhang 2006, for review). For example, the spectra of LGRBs are softer than that of SGRBs in general. Also, the pulse profiles of SGRBs are on average more symmetric than those of LGRBs (Zhang \& Xie 2007). The current Swift observations show that LGRBs have their median cosmological redshift $z_{\mathrm{m}} \sim 2.0$ higher than that of SGRBs $z_{\mathrm{m}} \sim 0.4$. All these differences suggest that both LGRBs and SGRBs are distinct physical phenomena and are produced by model-independent emission engines (e.g., Balázs et al. 2003). However, it is not clear what causes these differences, especially in the origin of the bimodal $T_{90}$ distribution.

Koshut et al. (1996) pointed out that the observed duration distribution may vary with instrument. It is therefore necessary to investigate if a new GRB class exists and/or what physical factors produce such properties (Gehrels et al. 2004). We focus our study on the related issues of GRB classification using the updated Swift data ${ }^{1,2,3}$.

\section{Observation and data selection}

The higher sensitivity and angular resolution of Swift make it superior to previous space telescopes (e.g. BATSE, BeppoSAX and HETE-2). Accurate follow-up observations have further added to its advantages (Gehrels et al. 2004). It can detect on an average about 2 GRBs per week within a 2 sr field of view, which is about two times more than that of pre-Swift missions (Mészáros 2006). However, the detection rate of SGRBs to total GRBs is much lower $(\sim 8 \%)$ than the rate by BATSE $(\sim 18 \%)$, which is attributed to both their different energy responses and the relatively high spectral hardness of SGRBs (e.g., Band 2006a,b; Gehrels et al. 2007). On the other hand, the lower sensitivity to short duration bursts of Swift, relative to BATSE, makes it accumulate relatively lower counts, comparable with the number of background counts (Band 2006a). The effect of the instrument may cause the detection rate of SGRBs to be somewhat underestimated.

In order to study the intrinsic properties of GRBs, we selected six data sets, namely s1-s6, as listed in Table 1. As of 2007 July 1, Swift has detected 75 LGRBs (s1) and 20 SGRBs (s2) with known duration and redshift. The $E_{\mathrm{p}}$ in the

\footnotetext{
${ }^{1}$ http://swift.gsfc.nasa.gov/docs/swift/archive/grb_ table.html

${ }^{2}$ http://grad40.as.utexas.edu/tour.php (GRBlog site).

${ }^{3}$ http://www.mpe.mpg.de/ jcg/grbgen.html
} 
Table 1. GRB samples of known durations $\left(T_{90}\right)$, redshifts $(z)$ and/or peak energies $\left(E_{\mathrm{p}}\right)$.

\begin{tabular}{ccclr}
\hline \hline Sample & No. & Class & Parameters & Ref. \\
\hline${\text { s } 1^{\dagger}}^{\dagger}$ & 75 & LGRBs & $T_{90} ; z$ & $1 ; 1 ;-$ \\
s2 $^{\dagger}$ & 20 & SGRBs & $T_{90} ; z$ & $1 ; 1 ;-$ \\
s3 $^{\dagger}$ & 44 & LGRBs & $T_{90} ; z ; E_{\mathrm{p}}$ & $1 ; 1 ; 4$ \\
s4 $^{\dagger}$ & 11 & SGRBs & $T_{90} ; z ; E_{\mathrm{p}}$ & $1 ; 1 ; 4$ \\
s5 $^{\ddagger}$ & 48 & LGRBs & $T_{90} ; z$ & 2,$3 ; 5 ;-$ \\
s6 $^{\sharp}$ & 18 & LGRBs & $T_{90} ; z$ & 2,$3 ; 5 ;-$ \\
\hline
\end{tabular}

Note: the references are given in order for $T_{90}, z$ and $E_{\mathrm{p}}$. Data sets: $\dagger$ Swift; $¥$ pre-Swift; $\sharp$ BATSE. Of the 20 SGRBs, GRB 050709, is adopted from HETE-2. Reference: 1. Swift public webpage; 2. Ghirlanda, Ghisellini \& Lazzati (2004); 3. GRBlog website; 4. Butler et al. (2007); 5. Friedman \& Bloom (2005).

observed $v F_{v}$ energy spectra are chosen to characterize the spectral hardness relations with duration. Here, 44 GRBs from s1 also have available $E_{\mathrm{p}}$ values and constitute our sample s3. In s2, only 11 sources have a measured $E_{\mathrm{p}}$ and these are employed to build the sample s4. Unfortunately, the remaining 9 bursts in s2 (i.e., GRB 050202, GRB 050906, GRB 050925, GRB 051105A, GRB 051210, GRB 060313, GRB 070209, GRB 070406 and GRB 070429B) do not have the measured redshifts. For these we assigned a redshift value of $z=0.5$ to the 9 bursts, approaching the median redshift of $z=0.4$, as assumed by Norris \& Bonnell (2006). These sources were included in the present study to improve the statistics. However, we found that the choice between $z=0.5$ and $z=0.4$ contributes only a small relative error of $\sim 0.07$, implying that the final results are not sensitive to the above assumed redshift values. In our fifth sample set, s5, we include 48 pre-Swift LGRBs whose $z$ and $T_{90}$ are already measured, in which 18 sources, less than half $(\sim 38 \%)$ of the 48 preSwift bursts, are detected by the BATSE mission and constitute our sample s6.

\section{Results}

\subsection{Distributions of durations in the Swift era}

To check whether the distributions of $T_{90}$ are significantly different, indicative of a dependence on the on-board instruments, we first obtained the distributions of Swift bursts in both observer and source frames. Then, we compare the durations of LGRBs between Swift and pre-Swift/BATSE missions.

\subsubsection{Observed $T_{90}$ distribution}

The accuracy of $T_{90}$ measurements is in principle affected by several factors including the identification of the time interval of a burst, the sensitivity of instrument, background modeling, the time resolution of the data, and the detailed shape of the burst time profile, etc. Fig. 1 shows the $T_{90}$ distribution for the 95 Swift GRBs, which include s1 and s2 samples (see Table 1). The best fit with a two-lognormal function gives the center values $\left(T_{90, p 1}=0.28_{-0.09}^{+0.14} \mathrm{~s}\right.$ and $\left.T_{90, p 2}=42.83_{-4.45}^{+4.60} \mathrm{~s}\right)$ and the widths $\left(w_{1}=19.05_{-11.11}^{+24.60} \mathrm{~s}\right.$ and $\left.w_{2}=18.20_{-3.41}^{+4.19} \mathrm{~s}\right)$ with the reduced Chisquare $\chi^{2} /$ d.o.f. $=0.67$, which are roughly consistent with those calculated from the BATSE data (McBreen et al. 1994; Meegan et al. 1996; Paciesas et al. 1999; Horváth 2002; Nakar 2007). Note that the number of objects classified as belonging to the two log-normals to itself has been allowed to be a free parameter. The superposed function has a minimum around $2 \mathrm{~s}$ as found



Fig. 1. Bimodal distribution of durations for the 95 GRBs (s1 and s2; histogram) and two-component log-normal fit to the data (solid line). The GRBs are divided into two classes at $T_{90} \simeq 2 \mathrm{~s}$ (vertical line): LGRBs (dotted line) and SGRBs (dashed line).

by Kouveliotou et al. (1993), indicating that the Swift sources are also divided into two classes, SGRBs and LGRBs, although the Swift is more sensitive to long soft bursts than the BATSE (Band 2006a,b; Gehrels et al. 2007). This is an interesting result since the different detectors with different bandwidth sensitivity do not affect the classification of GRBs in terms of duration.

\subsubsection{Intrinsic $T_{90}$ distribution}

One of the great progresses made by Swift is the increase in number of higher redshift sources. It is known that the median redshift of Swift LGRBs, $z_{\mathrm{m}} \sim 2$, is roughly two times larger than that of pre-Swift. As pointed out by previous authors (Bagoly et al. 2006; Jakobsson et al. 2006), the difference of redshift distributions between the two samples is statistically significant. This may lead to an evident discrepancy between the two intrinsic $T_{90}$ distributions. We therefore utilize the Swift sources to explore this possibility.

The potential spectrum evolution, as a result of cosmological redshift, can usually cause high energy $\gamma$ photons to shift into or out of the finitely sensitive bandwidth of a given detector. Note that here we had neglected the effect of a burst's spectrum itself softening with time (Ford et al. 1995). In this case, the transformation of $T_{90}$ from observer frame to source frame is generally expressed as $T_{90, \text { int }}=T_{90} /(1+z)^{\omega}$, in which $\omega=0.6$ or 1 , corresponding to energy stretching or not (Fenimore \& Bloom 1995; Mészáros \& Mészáros 1995; Mészáros \& Mészáros 1996; Horváth et al. 1996; Balázs et al. 2003). We consider a simple case of $\omega=1$ throughout this work, in which the intrinsic duration is given by $T_{90, \text { int }}=T_{90} /(1+z)$. We calculate the intrinsic duration $\left(T_{90, \text { int }}\right)$ distribution for the $95 \mathrm{GRBs}$ and compare it with the observed one. As shown in Fig. 2 , the $T_{90 \text {,int }}$ has a bimodal distribution and is significantly shifted toward shorter durations than the observed one. The best fit with a two-lognormal function gives two centers $\left(T_{90, p 1}=0.13_{-0.05}^{+0.12} \mathrm{~s}, T_{90, p 2}=12.30_{-1.83}^{+2.15} \mathrm{~s}\right)$ and two widths $\left(w_{1}=10.96_{-8.14}^{+31.69} \mathrm{~s}\right.$ and $\left.w_{2}=17.38_{-5.63}^{+8.32} \mathrm{~s}\right)$ with $\chi^{2} /$ d.o.f. $=0.92$, indicating that the distribution of $T_{90 \text {,int }}$ is indeed bimodal but systematically narrower and shifted towards low values of duration in comparison to the observed one. A Kolmogorov-Smirnov (K-S) test returns the statistic $D=0.27$ with a probability of $P=0.001$, suggesting the intrinsic and observed duration distributions are drawn from different parent 


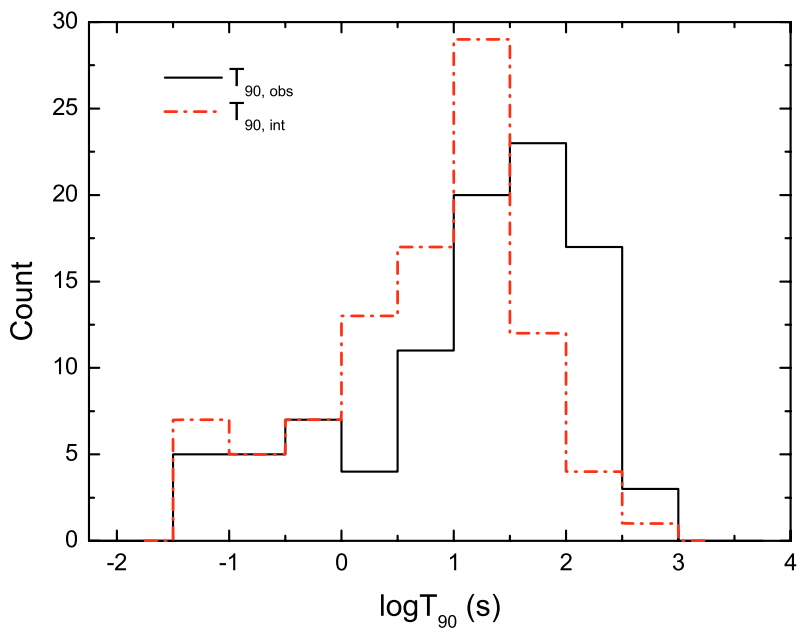

Fig. 2. Comparison between the observed (solid line the same as Fig. 1) and the intrinsic (dashed-dotted line) $T_{90}$ distributions.

populations. This result is consistent with the theoretical prediction by Bromm \& Loeb (2002), where they assumed that the formation of all GRBs tightly follows the cosmic star formation history. However, some instrument-dependent factors such as selection effects and discrepancy between different detectors can distort the resulting parent distribution of $T_{90, \text { int }}$. Once the disturbances are removed from the observed distribution, the remainder is the true parent distribution correlated with some physical predictions. In this case, our current result can offer some corroborative statistical evidence that the rate of GRBs may really trace the star formation history, partly because the redshifts of SGRBs had not been measured before Swift.

\subsection{3. $T_{90}$ of LGRBs: Swift vs. pre-Swift}

$T_{90}$ may be wrongly estimated because of the factors pointed out in Sect. 3.1.1. In particular, the measured duration has been found to be instrument-dependent (e.g., Koshut et al. 1996). We examined the dependence of $T_{90}$ on the instruments by comparing the normalized distributions of different detectors in Fig. 3. For this comparison, three samples including s1, s5, and s6 are used.

A log-normal fit to the six distributions gives the parameters listed in Table 2. We find that the Swift sample has a wider duration distribution than the pre-Swift and BATSE did in both observer and source frames, while the widths of preSwift and BATSE distributions of duration are equal. This implies that Swift can detect GRBs over a wider dynamic range of $T_{90}$, i.e., the fraction of longer and shorter Swift LGRBs is significantly higher than that of pre-Swift/BATSE LGRBs, except for the increased detection rate of LGRBs due to the higher sensitivity to long soft bursts of BAT loaded on Swift (Band 2006a,b). Considering the fact that Swift bursts can still be separated at $T_{90}=2 \mathrm{~s}$, we predict that the width of the $T_{90}$ distribution of Swift SGRBs could be equally wider as pre-Swift detectors. Furthermore, we see from Table 2 that the distribution centers of $T_{90, \mathrm{obs}}$ are very close to each other, while for the $T_{90 \text {,int }}$ distribution, the centers of the pre-Swift and BATSE bursts are much closer but significantly larger than that of Swift bursts. The K-S test of the pre-Swift and BATSE bursts provides the very large probability of $P \sim 0.99$ in both observer and source frames showing that the s5 and s6 samples are consistent with being drawn from the same parent distribution. For the Swift


Fig. 3. The normalized duration distributions for the Swift (s1; dashed line), pre-Swift (s5; solid line) and BATSE (s6; dotted line) LGRBs in the observer (upper panel) and the source (lower panel) frames.

$T_{90 \text {,int }}$ distribution, the obvious decrease of the center value is attributable to the relative increase of the fraction of high redshift sources, which could be caused by the ubiquitous threshold effect of different instruments (e.g., Bromm \& Loeb 2002; Band 2006a,b).

\subsection{Spectral hardness relation}

In theory, the peak energy $E_{\mathrm{p}}$ can be used to reflect the photon components, similar to the hardness ratio between different energy channels. The intrinsic peak energy $\left(E_{\mathrm{p}, i}\right)$ in the source frame is related to the $E_{\mathrm{p}}$ in the observer frame by $E_{\mathrm{p}, i}=E_{\mathrm{p}}(1+z)$. To verify the relation of spectral hardness to duration, we drew plots of peak energy versus $T_{90, \text { obs }}$ for the samples s3 and s4 as shown in Figs. $4 \mathrm{~b}$ and c. A linear correlation analysis gives the coefficient of $r=-0.27$ with probability $P=0.07$ for Fig. $4 \mathrm{~b}$ and $r=0.004$ with $P=0.98$ for Fig. 4c, indicating that the tendency towards SGRBs relatively harder than LGRBs exists in the observer frame and almost disappears in the source frame. The former accords with previous findings, for example, using the BATSE data (e.g., Kouveliotou et al. 1993) or the Swift data (e.g., Band 2006a).

We also compare the peak energy of LGRBs and SGRBs in both observer (Fig. 4a) and source frames (Fig. 4d). More cosmological redshift correction is required for LGRBs when we convert the physical variables from the observer frame to the source frame. As a result, the trend of the relative spectral softness of LGRBs (or the relative hardness of SGRBs) weakens. 
Table 2. The best-fit parameters to the three data sets.

\begin{tabular}{ccccccc}
\hline \hline & & $\log T_{90, \text { obs }}$ & & & $\log T_{90, \text { int }}$ & \\
Sample & $\mu$ & $w$ & $\chi^{2} /$ d.o.f. & $\mu$ & $w$ & $\chi^{2} /$ d.o.f. \\
\hline s1 & $1.57 \pm 0.04$ & $1.31 \pm 0.08$ & 2.4 & $1.06 \pm 0.04$ & $1.23 \pm 0.09$ & 2.8 \\
s5 & $1.63 \pm 0.04$ & $0.90 \pm 0.09$ & 2.5 & $1.32 \pm 0.03$ & $0.89 \pm 0.05$ & 0.9 \\
s6 & $1.62 \pm 0.04$ & $0.76 \pm 0.07$ & 0.5 & $1.29 \pm 0.03$ & $0.92 \pm 0.07$ & 0.3 \\
\hline
\end{tabular}

Note: the fitted parameters for the distribution center $(\mu)$ and the width $(w)$ are given in a logarithmic scale.
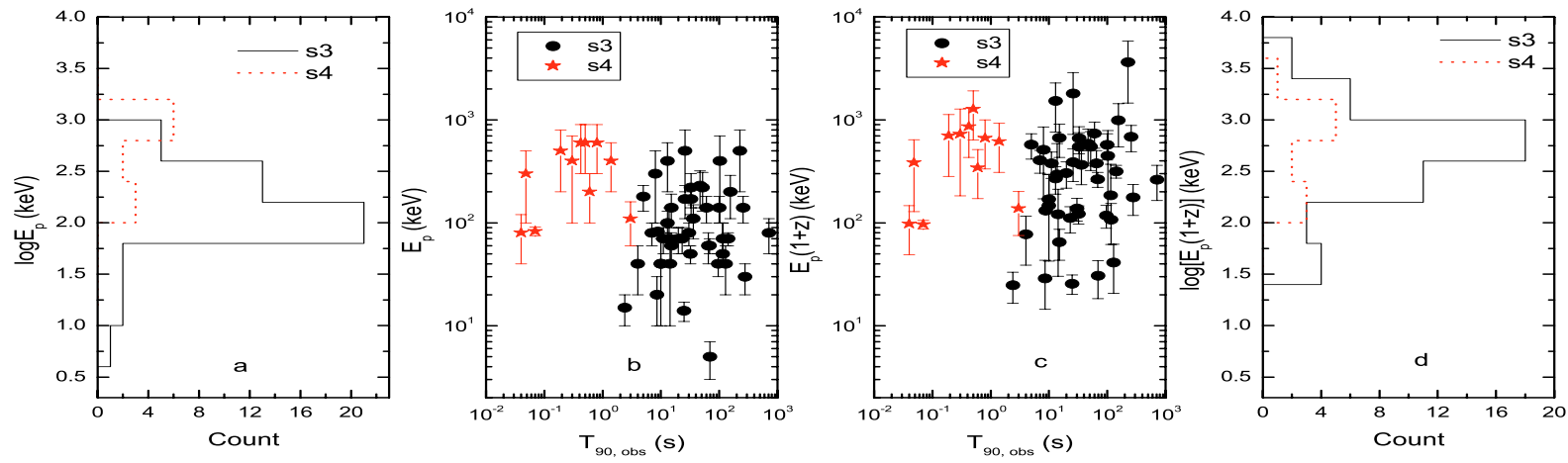

Fig. 4. Relations of $T_{90}$ with $E_{\mathrm{p}}$ (panel b)) and $E_{\mathrm{p}, i}$ (panel c)). Panels a) and d) show the distributions of the $E_{\mathrm{p}}$ and $E_{\mathrm{p}, i}$ in logarithmic timescale, respectively.

For our samples, the long and short bursts have the median values of $74_{-45}^{+120} \mathrm{keV}$ and $398_{-220}^{+493} \mathrm{keV}$, respectively, in the observer frame, and $302_{-202}^{+631} \mathrm{keV}$ and $617_{-365}^{+896} \mathrm{keV}$ in the source frame. A $\mathrm{K}-\mathrm{S}$ test returns the different probabilities of $P=0.01$ for Fig. $4 \mathrm{a}$ and $P=0.15$ for Fig. $4 \mathrm{~d}$, which suggests that the $E_{\mathrm{p}, i}$ of long and short bursts may be drawn from the same parent population.

\section{Discussion}

The duration distribution from the third BATSE catalog is suggested to have a three-component log-normal form (Horváth 1998; Mukherjee 1998; Hakkila et al. 2000). Similarly, the duration distribution of the current $4 \mathrm{~B}$ catalog $^{4}$ shows the possible existence of an intermediate group. Further studies show that the third group is either the excess of SGRBs with low fluence (Hakkila et al. 2003) or the softest LGRBs (Horváth et al. 2006). It is still controversial whether the third class exists or not, due to the lack of a physical explanation (Horváth et al. 2006; see however, Chattopadhyay et al. 2007). The third group might be caused by an instrumental bias that reduces the durations of faint LGRBs (e.g., Hakkila et al. 2000; Hakkila et al. 2003), suggesting that their presence is not physical but phenomenological. Therefore, the bimodality of the duration distribution is widely accepted today.

What causes the bimodality is an interesting but unsolved problem owing to the absence of direct observational evidence, although several works have tried to explain the bimodality within different scenarios. It was suggested that the different spin axes of millisecond pulsars can explain the two GRB classes (Usov 1992; Yi \& Blackman 1998). Subsequently, Huang et al. (2003) studied the neutron star kick model (Dar \& Plaga 1999) in detail and proved that this model can successfully account for the two log-normal distributions if the central engine has a neutron star of a high kick velocity greater than $\sim 1000 \mathrm{~km} \mathrm{~s}^{-1}$.

\footnotetext{
${ }^{4}$ http://www.batse.msfc .nasa.gov/batse/grb/duration/
}

Yamazaki et al. (2004) put forward the so-called unified model consisting of multiple subjets within an inhomogeneous main jet. Using this model, Toma et al. (2005) explained that the bimodality originates from discrete emitters in the main jet. They also predicted that the two kinds of bursts should have the same origin, i.e., supernovae, instead of the leading models, which predict that the LGRBs and SGRBs are produced due to the core collapse of a massive star and the merger of double compact objects, respectively (see e.g., Cheng \& Lu 2001; Zhang \& Mészáros 2004; Piran 2005; Mészáros 2006; and Lee \& Ramirez-Ruiz 2007, for reviews). However, further investigations to the unified model showed that the bimodal distribution could be reproduced only for some special parameters (Janiuk et al. 2006).

Recently, from an independent analysis of distinct timescales, Zhang et al. (2007) suggested that LGRBs occur at greater distances from the central engine while SGRBs at smaller distances, i.e., two distinct $\gamma$-ray emission regions may result in two different properties of GRBs, including the varieties of pulse profiles, as mentioned above. They also pointed out the fact that LGRBs usually have a long positive spectral lag (e.g., Norris et al. 2000; Daigne \& Mochkovitch 2003; Chen et al. 2005) and SGRBs have negligible lag (Norris \& Bonnell 2006; Zhang et al. 2006). This can be explained under the assumption that the curvature effect is a main contributor to the spectral lag (Ryde 2005; Shen et al. 2005). The predication of distinct emission regions for different GRB classes and/or the nature of the bimodality still needs to be clarified with more accurate observations in the future.

\section{Conclusions}

- We find that the observed durations of Swift GRBs have twolognormal distributions divided clearly at $T_{90} \simeq 2 \mathrm{~s}$. This implies that the classification in terms of duration is unchanged from pre-Swift to the Swift era. 
- The intrinsic durations also show a bimodal distribution but shifted systematically toward the smaller value and the distribution exhibits a narrower width relative to the observed distribution.

- The $T_{90, \text { int }}$ distributions of Swift and pre-Swift/BATSE LGRBs are significantly different in the width and center values.

- Swift bursts have a wider duration dynamic range than preSwift and BATSE bursts.

- The spectra of the SGRBs are predominantly harder than the LGRBs, confirming the previous results from the BATSE bursts. However, the trend of LGRBs with a relatively softer spectrum largely weakens in the source frame.

Acknowledgements. We thank David L. Band and Attila Mészáros for their helpful comments and suggestions. The authors thank the anonymous referee for a thorough and constructive report which led to a substantial improvement of the paper. We thank Istvan Horváth for good communication and G. Maheswar for critical reading of the manuscript. Z.B.Z. wishes to acknowledge the postdoctoral fellowship from the Korea Astronomy and Space Science Institute (KASI).

\section{References}

Band, D. L. 2006a, ApJ, 644, 378

Band, D. L. 2006b, AIPC, 836, 704

Balázs, L. G., Bagoly, Z., Horváth, I., Mészáros, A., \& Mészáros, P. 2003, A\&A, 401, 129

Bagoly, Z., Mészáros, A., Balázs, L. G., et al. 2006, A\&A, 453, 797

Bromm, V., \& Loeb, A. 2002, ApJ, 575, 111

Butler, N. R., Kocevski, D. B., Joshua, S., et al. 2007, ApJ, 671, 656

Chattopadhyay, T., Misra, R., Chattopadhyay, A. K., \& Naskar, M. 2007, ApJ, 667, 1017

Chen, L., Lou, Y.-Q., Wu, M., et al. 2005, ApJ, 619, 983

Cheng, K. S., \& Lu, T. 2001, ChJAA, 1, 1

Daigne, F., \& Mochkovitch, R. 2003, MNRAS, 342, 587

Dar, A., \& Plaga, R. 1999, A\&A, 349, 259

Fenimore, E. E., \& Bloom, J. S. 1995, ApJ, 453, 16
Fishman, G. J. 1999, A\&AS, 138, 395

Ford, L. A., Band, D. L., Matteson, J. L., et al. 1995, ApJ, 439, 307

Friedman, A. S., \& Bloom, J. S. 2005, ApJ, 627, 1

Gehrels, N., Chincarini, G., Giommi, P., et al. 2004, ApJ, 611, 1005

Gehrels, N., Cannizzo, J. K., \& Norris, J. P. 2007, NJPh, 9, 37

Ghirlanda, G., Ghisellini, G., \& Lazzati, D. 2004, ApJ, 616, 331

Hakkila, J., Haglin, D. J., Pendleton, G. N., et al. 2000, ApJ, 538, 165

Hakkila, J., Giblin, T. W., Roiger, R. J., et al. 2003, ApJ, 582, 320

Horváth, I. 1998, ApJ, 508, 757

Horváth, I. 2002, A\&A, 392, 791

Horváth, I., Mészáros, P., \& Mészáros, A. 1996, ApJ, 470, 56

Horváth, I., Bala'zs, L. G., Bagoly, Z., et al. 2006, A\&A, 447, 23

Huang, Y. F., Dai, Z. G., Lu, T., Cheng, K. S., \& Wu, X. F. 2003, ApJ, 594, 919

Jakobsson, P., Levan, A., Fynbo, J. P. U., et al. 2006, AIPC, 836, 552

Janiuk, A., Czerny, B., Moderski, R., et al. 2006, MNRAS, 365, 874

Katz, J. I., \& Canel, L. M. 1996, ApJ, 471, 915

Kawai, N., et al. 2005 [arXiv: astro-ph/0512052]

Kouveliotou, C., Meegan, C. A., Fishman, G. J., et al. 1993, ApJ, 413, L101

Koshut, T. M., Paciesas, W. S., Kouveliotou, C., et al. 1996, ApJ, 463, 570

Lee, W. H., \& Ramirez-Ruiz, E. 2007, NJPh, 9, 17

Mao, S., Narayan, R., \& Piran, T. 1994, ApJ, 420, 171

McBreen, B., Hurley, K. J., Long, R., et al. 1994, MNRAS, 271, 662

Meegan, C. A., Pendleton, G. N., Briggs, M. S., et al. 1996, ApJS, 106, 65

Mészáros, A., \& Mészáros, P. 1996, ApJ, 466, 29

Mészáros, P. 2006, RPPh, 69, 2259

Mészáros, P., \& Mészáros, A. 1995, ApJ, 449, 9

Mukherjee, S., Feigelson, E. D., Jogesh, B. G., et al. 1998, ApJ, 508, 314

Nakar, E. 2007, PhR, 442, 166

Norris, J. P., \& Bonnell, J. T. 2006, ApJ, 643, 266

Paciesas, W., Meegan, C., Pendleton, G., et al. 1999, ApJS, 122, 465

Piran, T. 2005, Rvmp, 76, 1143

Ryde, F. 2005, ApJ, 429, 869

Shen, R. F., Song, L. M., \& Li, Z. 2005, MNRAS, 362, 59

Toma, K., Yamazaki, R., \& Nakamura, T. 2005, ApJ, 620, 835

Usov, V. V. 1992, Nature, 357, 472

Yamazaki, R., Ioka, K., \& Nakamura, T. 2004, ApJ, 607, L103

Yi, I., \& Blackman, E. G. 1998, ApJ, 494, L163

Zhang, B. 2006, Nature, 444, 1010

Zhang, B., \& Mészáros, P. 2004, IJMPA, 19, 2385

Zhang, Z. B., \& Xie, G. Z. 2007, Ap\&SS, 310, 19

Zhang, Z. B., Xie, G. Z., Deng, J. G., et al. 2006, MNRAS, 373, 729

Zhang, Z. B., Xie, G. Z., Deng, J. G., et al. 2007, AN, 328, 99 